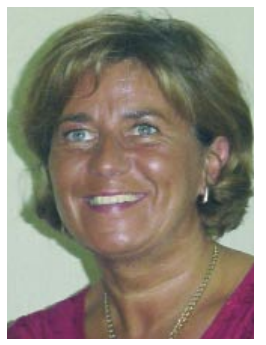

Heike Beneš

\section{Was gibt es Neues zum Restless-legs-Syndrom?}

Heike Beně̌

somni bene

Institut für Medizinische Forschung und Schlafmedizin Schwerin

psychoneuro 2004; 30 (8): 438-443
Die RLS-Forschung hat sich in den letzten Jahren dynamisch entwickelt. Dieser Beitrag gibt einen Überblick zum aktuellen Stand der Klinik, Epidemiologie, Pathophysiologie, Diagnostik, Klassifikation sowie der Therapie des RLS. Eine Prävalenz des RLS von ca. 10\% der erwachsenen Bevölkerung der westlichen Länder wurde mehrfach bestätigt. Im Bereich der Diagnostik sind revidierte Diagnosekriterien für das RLS und die Entwicklung von standardisierten Methoden zur Erfassung der RLS-Diagnose und des Schweregrades herauszustellen. Neue klinische Studien belegen die Wirksamkeit dopaminerger Medikamente (L-Dopa, Dopaminagonisten) beim RLS. Augmentation, insbesondere der frühere Beginn der Beschwerden am Tage, kann jedoch Therapieumstellungen erzwingen. Therapiealternativen sind Opiate und Gabapentin.

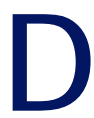
as RLS gehört mit einer Prävalenz von ca. $10 \%$ in der europäischen und nordamerikanischen Bevölkerung zu den häufigsten neurologischen Erkrankungen und wird unverständlicherweise bis heute leider oft nicht erkannt oder fehldiagnostiziert. In einer epidemiologischen Studie in der deutschen Bevölkerung bei >65-Jährigen ergab sich eine Prävalenz von $9,8 \%$ (14), wobei Frauen mit 13,8\% doppelt so häufig wie Männer (6,1\%) betroffen waren. Diese Daten wurden kürzlich in einer epidemiologischen Studie in Vorpommern bestätigt: in einer für die Bevölkerung repräsentativen Stichprobe von 4310 Befragten im Alter zwischen 20 und 79 Jahren betrug die Prävalenz 10,6\%, Frauen waren doppelt so häufig betroffen wie Männer, die Prävalenz stieg mit dem Alter an, bei Frauen mit der Anzahl von Schwangerschaften (6).
Nicht selten können Patienten, die unter einem Restless-legs-Syndrom leiden, ihre Beschwerden nur schwer beschreiben und klagen vordergründig über z.T. schwere Schlafstörungen durch unruhige Beine und häufige nächtliche Wachzeiten. Die quälenden Missempfindungen der Beine, die mit einem unstillbaren Bewegungsdrang einhergehen und typischerweise fast ausschließlich in Ruhesituationen, ganz besonders heftig abends im Bett oder nachts auftreten, zwingen die Betroffenen, sich zu bewegen oder nächtelang umherzulaufen. Die meisten Patienten können ihre Beschwerden durch Bewegung und körperliche Aktivität lindern, in der Regel aber nur so lange, wie die Bewegung anhält. Nun ist es jedoch unmöglich, ständig in Bewegung zu bleiben. Die Ausprägung der Symptomatik folgt einer zirkadianen Rhythmik, die sich umgekehrt proportional zur Körper- temperaturkurve verhält und somit zu einer Zunahme der Beschwerden am Abend bis kurz nach Mitternacht führt. Bei über $90 \%$ der Betroffenen gehen damit erhebliche Ein- und Durchschlafstörungen mit resultierender Tagesmüdigkeit und Erschöpfung einher, die nicht selten der Grund für die erste Konsultation eines Arztes sind. Gerade die durch hartnäckige Schlafstörungen erschöpften und verzweifelten Patienten sehnen sich nach einer Zeit der Ruhe und Entspannung. Durch Massagen oder Bürsten der Beine, kalte Fußbäder oder Güsse, Kniebeugen oder Fahrradfahren lassen sich manche Abende und Nächte überstehen.

\section{Diagnostik des RLS}

Basis der Diagnostik ist eine ausführliche und gezielte Anamnese. Zur eindeutigen Diagnose müssen folgende vier Minimalkriterien des RLS erfüllt sein (International RLS Study-Group (IRLSSG)) (3):

- Bewegungsdrang der Beine (eventuell auch der Arme), meistens in Verbindung mit unangenehmen Missempfindungen der betroffenen Extremität(en)

- Auftreten bzw. Verstärkung dieser Beschwerden in Ruhesituationen

- Besserung bzw. Beseitigung der Beschwerden durch Bewegung 
- Zunahme der Beschwerden abends oder nachts.

Unterstützende Kriterien beinhalten ein Ansprechen auf dopaminerge Behandlung mit Linderung des Bewegungsdranges und der Missempfindungen, eine positive Familienanamnese und periodische Beinbewegungen im Schlaf (PLMS) und im Wachen (PLM) in der Polysomnographie. PLM sind definiert als mindestens vier aufeinanderfolgende Bewegungen von 0,5-5 sec Dauer in Intervallen von 5-90 sec, sie können uni- oder bilateral, simultan oder alternierend auftreten und sind häufig mit einer Dorsalflexion des Sprunggelenkes bzw. der Großzehe verbunden.

Zusätzliche Kriterien wie ein unauffälliger neurologischer Befund (beim idiopathischen RLS), ein meist initial fluktuierender, später progredienter klinischer Verlauf und Schlafstörungen können die Diagnose erhärten.

Zum Ausschluss sekundärer RLSFormen sind Laboruntersuchungen (Blutbild, Kreatinin, Eisen, Ferritin, Blutzucker, TSH) sowie bei Verdacht auf eine auslösende bzw. zusätzlich bestehende Polyneuropathie eine EMG/ENG-Untersuchung zu empfehlen.

Eine polysomnographische $\mathrm{Ab}$ klärung des RLS ist besonders bei Zweifeln an der Diagnose, therapierefraktären RLS-Patienten, Kindern und Jugendlichen vor einer geplanten dopaminergen Therapie und dem Verdacht auf zusätzlich bestehende schlafspezifische Störungen (Schlaf-Apnoe-Syndrom, Narkolepsie) anzuraten.

Kürzlich wurde von der Autorin ein Algorithmus für die Diagnosefindung des RLS entwickelt: der RLSDiagnose-Index (RLS-DI, Tab. 1). Er bezieht alle aus der klinischen Erfahrung wichtigen diagnostischen Informationen mit annähernd gleicher Wertigkeit ein. Die Gewichtung einzelner Symptome, besonders die diagnose-kritische Bedeutung des Fehlens spezifischer Symptome, variiert in Abhängigkeit von der Intensität und der Relevanz der Symptome für die RLS-Diagnose. Er erlaubt eine Aussage zur Wahrscheinlichkeit der RLS-

\section{Tab. 1 Der Restless Legs Syndrom Diagnose-Index (RLS-DI)}

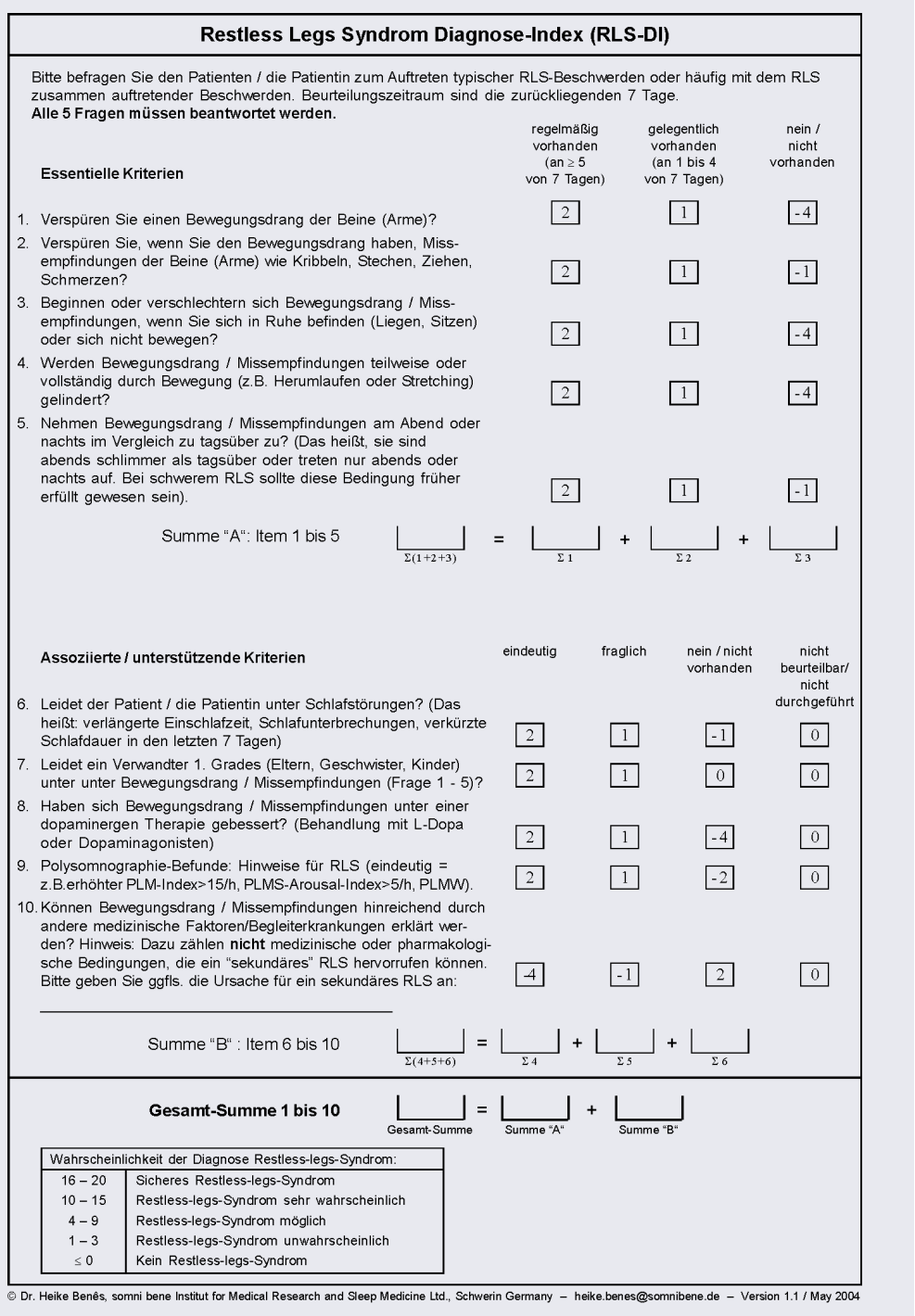

Diagnose nach den jeweils vorliegenden anamnestischen und diagnostischen Informationen und wird derzeit in klinischen Studien validiert.

Zur gezielten Bewertung der Ausprägung des RLS wurden inzwischen mehrere Schweregradskalen entwickelt: die IRLS-Rating-Scale (von der IRLSSG entwickelt und validiert, 9) (Tab. 2) umfasst zehn Fragen zu RLS-Beschwerden und ihre Auswirkungen auf Schlaf und Tagesbefindlichkeit/Lebensqualität, wobei jede Frage mit 0-4 (max. Ausprägung) beantwortet werden kann, so dass sich ein Gesamtscore von 0 (kein RLS)-40 (sehr schwere Ausprägung) Punkten ergibt.

Die RLS-6-Schweregradsskalen bestehen aus sechs Skalen, die vor- wiegend auf den Zeitpunkt des Auftretens der RLS-Beschwerden fokussiert sind (Schweregrad tagsüber, beim Einschlafen, nachts) und damit eine RLS-spezifische Aussage über die Ausprägung der Symptome zu verschiedenen Tageszeiten und deren Auswirkungen auf Schlaf und Tagesbefindlichkeit des Patienten zulassen (Tab. 3). Diese Skala eignet sich gut für Verlaufsbeobachtungen, z.B. um zu kontrollieren, wie gut ein Patient auf eine neu angesetzte Therapie anspricht (10).

\section{Klassifikation des RLS} des RLS:

- die idiopathische Form, die bei bis zu 50\% der Patienten gene-
Man unterscheidet zwei Formen 


\section{Tab. 2 IRLS-Beurteilungsbogen zum Restless-legs-Syndrom (Version 2.1)}

„In der letzten Woche...“

(vom Patienten zu beantworten, vom Untersucher zu dokumentieren)

1 Wie stark würden Sie die RLS-Beschwerden in Ihren Beinen oder Armen einschätzen? 4 = sehr, 3 = ziemlich, 2 = mäßig, 1 = leicht, 0 = nicht vorhanden

2 Wie stark würden Sie Ihren Drang einschätzen, sich wegen Ihrer RLS-Beschwerden bewegen zu müssen?

4 = sehr, 3 = ziemlich, 2 = mäßig, 1 = leicht, 0 = nicht vorhanden

3 Wie sehr wurden die RLS-Beschwerden in Ihren Beinen oder Armen durch Bewegung gelindert?

4 = überhaupt nicht gelindert, 3 = ein wenig gelindert, 2 = mäßig gelindert, 1 = vollständig oder fast vollständig gelindert, 0 = es mussten keine RLS-Beschwerden gelindert werden

$4 \quad$ Wie sehr wurde Ihr Schlaf durch Ihre RLS-Beschwerden gestört? 4 = sehr, 3 = ziemlich, 2 = mäßig, 1 = leicht, 0 = überhaupt nicht

$5 \quad$ Wie müde oder schläfrig waren Sie tagsüber wegen Ihrer RLS-Beschwerden? 4 = sehr, 3 = ziemlich, 2 = mäßig, 1 = leicht, 0 = überhaupt nicht

$6 \quad$ Wie stark waren Ihre RLS-Beschwerden insgesamt? 4 = sehr, 3 = ziemlich, 2 = mäßig, 1 = leicht, 0 = nicht vorhanden

$7 \quad$ Wie oft sind Ihre RLS-Beschwerden aufgetreten? 4 = sehr oft (das heißt an 6 bis 7 Tagen in der Woche), 3 = oft (das heißt an 4 bis 5 Tagen in der Woche), 2 = manchmal (das heißt an 2 bis 3 Tagen in der Woche), 1 = selten (das heißt an einem Tag in der Woche), 0 = überhaupt nicht

8 Wenn Sie RLS-Beschwerden hatten, wie stark waren diese durchschnittlich? 4 = sehr (das heißt an 8 Stunden oder mehr an einem 24-Stundentag), 3 = ziemlich (das heißt an 3 bis 8 Stunden an einem 24-Stundentag), 2 = mäßig (das heißt an 1 bis 3 Stunden an einem 24-Stundentag), 1 = leicht (das heißt an weniger als 1 Stunde an einem 24Stundentag), $0=$ nicht vorhanden

9 Wie sehr haben sich Ihre RLS-Beschwerden auf Ihre Fähigkeit ausgewirkt, Ihren Alltagstätigkeiten nachzugehen, z.B. ein zufriedenstellendes Familien-, Privat-, Schul- oder Arbeitsleben zu führen?

4 = sehr, 3 = ziemlich, 2 = mäßig, 1 = leicht, 0 = überhaupt nicht

10 Wie stark haben Ihre RLS-Beschwerden Ihre Stimmung beeinträchtigt, waren Sie z.B. wütend, niedergeschlagen, traurig, ängstlich oder gereizt?

4 = sehr, 3 = ziemlich, 2 = mäßig, 1 = leicht, 0 = überhaupt nicht

(c) International Restless Legs Study Group

Hinweis: Gesamtscore: 1-10 = leichte, 11-20 = mäßige, 21-30 = schwere, 31-40 = sehr schwere Ausprägung des RLS

tisch determiniert ist und familiär gehäuft auftritt und

- die symptomatische Form, die sich auf der Basis einer anderen Grunderkrankung entwickelt.

Beim sog. idiopathischen RLS kann keine auslösende Grunderkrankung diagnostiziert werden, die Häufigkeit einer genetischen Prädisposition wird bei dieser Form mit 40-80\% vermutet, wobei der Vererbungsgang als autosomal-dominant angegeben wird $(3,11)$.

Ein RLS kann bereits bei Kindern und Jugendlichen auftreten, wobei die RLS-Symptomatik in dieser Altersgruppe häufig als „Hyperaktivitätssyndrom“ oder „Wachstums- schmerzen“ verkannt wird. Zu den häufigsten symptomatischen Formen gehören das RLS bei Urämie, bei Eisenmangelanämie, rheumatischer Polyarthritis, in der Schwangerschaft und bei diversen neurologischen Erkrankungen (Polyneuropathien, Myelopathien, Spinalkanalstenose, M. Parkinson). Ein pharmakogen induziertes RLS wurde überwiegend bei dopaminantagonistisch wirkenden Substanzen, wie bei den klassischen, gelegentlich auch bei sog. „atypischen“ Neuroleptika wie Olanzapin, tri- und tetrazyklischen Antidepressiva, Serotonin-Wiederaufnahmehemmern und Metoclopramid beobachtet (17).

\section{- Pathophysiologie des RLS}

Die Pathophysiologie des RLS ist bisher noch nicht eindeutig geklärt, jedoch haben neurophysiologische, zirkadiane, pharmakologische und bildgebende Studien zum Verständnis der Erkrankung beigetragen.

Auf Grund der guten therapeutischen Wirksamkeit von dopaminergen und opioidergen Substanzen geht man von einer Beteiligung der entsprechenden Neurotransmittersysteme aus. Es sind bisher bei RLSPatienten keine strukturellen Veränderungen des zentralen Nervensystems beschrieben worden. In bildgebenden Untersuchungen mittels SPECT sowie PET-Technik fanden sich grenzwertig erniedrigte dopaminerge striatale Rezeptorbindungen, die auf eine Funktionsstörung im striatalen dopaminergen System hinweisen. Es könnte sich hierbei auch um sekundäre Phänomene handeln. In Untersuchungen mittels funktioneller Kernspintomographie zeigten sich Hinweise für eine Beteiligung von Hirnstamm- und Kleinhirnstrukturen beim Auftreten von motorischen RLS-Symptomen. Die wohl sehr komplexe Funktionsstörung verschiedener Neurotransmittersysteme führt nach neurophysiologischen Befunden zu einer gesteigerten Erregbarkeit mono- und polysynaptischer Reflexbögen auf der Ebene des Hirnstamms und des Rückenmarks und damit zu Enthemmungsphänomenen im absteigenden retikulären System. Auch eine Beteiligung des peripheren Nervensystems wird diskutiert. Inwieweit diese Strukturen an dem primären Auslösemechanismus - falls es diesen überhaut gibt - beteiligt sind, oder jeweils einen unterschiedlichen Beitrag bei der Entstehung der sensorischen und motorischen Komponente der Erkrankung haben, ist noch unklar $(2,17)$.

\section{Differentialdiagnostik des RLS}

Die häufigste und wichtigste Differentialdiagnose des RLS ist die Polyneuropathie (PNP). Erschwerend kommt hinzu, daß einige Patienten sowohl eine PNP als auch eine eindeutige RLS Symptomatik aufweisen, sodass diese Differentialdiag- 
nose problematisch sein kann und sich nicht immer ausschließt.

Auch Radikulopathien, Myelopathien und chronische Schmerzsyndrome müssen sorgfältig von einem RLS unterschieden werden.

\section{Behandlung des RLS}

Bei leichteren oder intermittierenden Formen können allgemeine Maßnahmen einer verbesserten Schlafhygiene, z.B. der Verzicht auf abendlichen Kaffeegenuß oder Alkohol, Wärme, schwere körperliche Arbeit und Reduktion von Stress schon hilfreich sein.

Die Indikation zu einer medikamentösen Therapie des Restlesslegs-Syndroms sollte sorgfältig gestellt sein: bei nur intermittierenden RLS-Beschwerden ( $<1 \mathrm{x}$ wöchentlich) oder nur geringer Beeinträchtigung der Schlafqualität ohne Einbußen der Tagesbefindlichkeit ist eine gezielte medikamentöse Behandlung nicht angezeigt. Führen jedoch die typischen Beschwerden sowie die Folgen hartnäckiger Schlafstörungen zu einer beeinträchtigten Tagesbefindlichkeit und Lebensqualität, sollte dieses Krankheitsbild gezielt medikamentös behandelt werden. Genaue Untersuchungen über den Anteil therapiebedürftiger RLS-Patienten liegen noch nicht vor, nach klinischen Erfahrungen jedoch sind mindestens $25 \%$ der bereits diagnostizierten RLS-Patienten behandlungsbedürftig.

Vor Beginn einer medikamentösen Therapie sollten folgende Bedingungen erfüllt sein:

- Die Diagnose Restless-legs-Syndrom muss zweifelsfrei gestellt worden sein

- Mögliche Grunderkrankungen, die ein sekundäres Restless-legsSyndrom auslösen können, müssen ausgeschlossen oder, wenn möglich, primär behandelt worden sein

- Bei Eisenmangel besteht die Therapieoption in der Substitution von Eisen. Für das urämische RLS ist beschrieben, dass die RLS-Beschwerden nach einer Nierentransplantation verschwanden (21)

- RLS-auslösende bzw. -verstärkende Medikamente (z.B. Neuro- leptika, tri- und tetrazyklische Antidepressiva) sollten ausgetauscht bzw. abgesetzt werden

- Die Lebensqualität ist durch die Symptomatik des RLS - und damit auch durch Schlafstörungen und Tagesmüdigkeit - so stark beeinträchtigt, dass ein erheblicher Leidensdruck besteht.

Ist bei einem symptomatischen/sekundären RLS eine kausale Therapie nicht möglich, so muss wie beim idiopathischen RLS eine gezielte Pharmakotherapie eingeleitet werden.

Ausgangspunkt für die medikamentöse Therapie waren die überzeugenden und auch überraschenden Ergebnisse von RLS-Studien 1986 (12, 15), die eine signifikante Therapieeffizienz von L-Dopa beim RLS nachweisen konnten. Seither ist durch umfangreiche klinische Studien und Anwendungsbeobachtungen die Wirksamkeit dopaminerger Substanzen beim RLS nachgewiesen worden.

Durch die spezifische und kurzfristig einsetzende Wirkung von LDopa/Decarboxylasehemmer auf die sensible und motorische RLS-Symptomatik wird derzeit der Einsatz eines sog. „L-Dopa-Tests“ als diagnostisches Kriterium ausgearbeitet (Stiasny et al., in Vorbereitung). Nach unseren Erfahrungen sprechen ca. 80-90\% aller RLS-Patienten schon nach einmaliger Gabe von 100 mg LDopa mit einer deutlichen Be-

\section{Tab. 3 RLS-6 Beurteilungsskalen}

Bitte beantworten Sie (der Patient) die folgenden Fragen für die letzten 7 Tage bzw. Nächte:

1 Wie zufrieden waren Sie mit Ihrem Schlaf in den letzten 7 Nächten? 0 = völlig zufrieden, ..., 10 = völlig unzufrieden

Wie schwer waren Ihre RLS-Symptome während der letzten 7 Nächte bzw. Tage in den folgenden Situationen?

2 Beim Einschlafen

3 Während der Nacht

4 Tagsüber in Ruhe (im Sitzen, im Liegen)

5 Tagsüber, wenn Sie nicht in Ruhe waren, sondern sich körperlich betätigt haben (Gehen, berufliche Aktivitäten, Hausarbeit, Freizeitaktivitäten)

Skala jeweils: 0 = nicht vorhanden, $1=$ sehr leicht, $\ldots, 10=$ sehr schwer

6 Wie müde oder schläfrig waren Sie in den letzten 7 Tagen tagsüber (zwischen Aufstehen und Schlafengehen)?

0 = überhaupt nicht, 1 = nur ein wenig, ..., 10 = sehr müde

(c) Kohnen R, Oertel WH, Stiasny-Kolster K, Beneš H, Trenkwalder C (2003).

Hinweis: Die Skalen werden einzeln ausgewertet, ein Gesamtscore ist nicht definiert. 
Dopa, um eine rasche Beschwerdelinderung zu erreichen. Da die Wirkung von L-Dopa-Standardpräparaten nur ca. 4-5 h anhält, ist bei zusätzlich auftretenden Durchschlafstörungen in der zweiten Nachthälfte eine Kombinationstherapie mit einem L-Dopa-Retard-Präparat erforderlich. Bei intermittierender RLS-Symptomatik auch tagsüber kann L-Dopa im Einzelfall auch mehrfach täglich in niedriger Dosierung (50-100 mg) verordnet werden, hierfür hat sich besonders die lösliche L-Dopa-Tablette (Madopar ${ }^{\circledR}$ LT) wegen ihres raschen Wirkungseintritts als „ad hoc“-Medikation sehr bewährt.

L-Dopa ist sowohl bei idiopathischem als auch urämischem RLS auch in der Langzeitbehandlung gut verträglich. Aus der Parkinson-Therapie bekannte und gefürchtete $\mathrm{Ne}$ benwirkungen wie Dyskinesien oder psychotische Symptome wurden bei RLS-Patienten nicht beobachtet. Die herausragenden Vorzüge der LDopa-Therapie beim RLS liegen im schnellen Wirkungseintritt und der guten Steuerbarkeit - auch bei intermittierender oder bedarfsgerecht angepasster Gabe.

Allerdings gibt es auch Probleme in der L-Dopa-Therapie des RLS: bei einem Teil der über längere Zeit mit L-Dopa behandelten RLS-Patienten kann es unter stabiler Therapie zu einer sog. „Augmentation“ oder einem „Time-shift“ der Symptomatik kommen, d.h. zu einer Zunahme bzw. Verschiebung der RLS-Symptomatik in den Tag hinein (1). Hierbei kommt es zu

- $\quad$ einer zeitlichen Vorverlagerung der RLS-Beschwerden

- einer Zunahme der Beschwerdeintensität

- einer Verkürzung der Ruhezeit vor dem Auftreten von RLSSymptomen, insbesondere am Tage und

- einer Ausdehnung der Symptomatik auf andere Körperteile (z.B. Arme).

Viele Patienten berichten dabei über einen Wirkverlust bzw. eine Toleranzentwicklung der Medikation. Dieses Phänomen der Augmentation wurde inzwischen auch bei einzelnen Dopaminagonisten in unterschiedlicher Ausprägung beobachtet: Pramipexol bis 32\% (20), Pergolid bis 27\% (16), Cabergolin bis 3\% (4), Ropinirol bis 2,6\% (8). Allerdings sind diese Augmentationsraten häufig aus einer Analyse von unerwünschten Ereignissen entnommen, spezifische Untersuchungen mit einem standardisierten Instrument (der Augmentation-SchweregradsRating Skala ASRS) werden derzeit durchgeführt. Höhere Häufigkeiten von Augmentation als oben angegeben können bei spezifischen Erhebungen mit der ASRS erwartet werden.

Seltener kann auch ein ReboundPhänomen, d.h. eine Vorverlagerung der Symptomatik in die frühen Morgenstunden bei nachlassender Wirkung der abendlichen dopaminergen Medikation auftreten.

Da es Hinweise dafür gibt, dass die Entwicklung dieser unerwünschten Phänomene dosisabhängig ist, sollte eine Maximaldosierung von $400 \mathrm{mg}$ L-Dopa/die nicht überschritten werden (19). Treten o.g. Phänomene im Verlauf einer LDopa-Therapie auf, sollte die Medikation auf einen länger wirksamen Dopaminagonisten umgestellt werden, da hierunter sowohl Augmentation als auch Time-shift-Phänomene i.d.R. innerhalb weniger Tage verschwinden.

\section{Dopaminagonisten}

Fast alle zugelassenen Dopaminagonisten wurden in den letzten Jahren in offenen und plazebo-kontrollierten Studien auf ihre Wirksamkeit bei RLS-Beschwerden untersucht und zeigen ähnlich positive Effekte sowohl auf die subjektiven RLS-Beschwerden als auch auf die Schlafqualität. Besonders Dopaminagonisten mit längeren Halbwertszeiten wie Cabergolin ( $>65$ h), Pergolid (15-42 h) und Pramipexol (8-12 h) garantieren eine kontinuierliche Wirkung über die gesamte Dauer der Nacht und werden i.d.R. in einer Einmaldosis am Abend verabreicht. Dabei liegt auch hier die notwendige Dosis deutlich niedriger (ca. um Faktor 2-4) als bei der Parkinson-Behandlung: für Pramipexol wird eine Dosierung von 0,18-0,5 mg zur Nacht empfohlen, in kontrollierten Multicenterstudien zu Cabergolin wurde eine optimale Dosis von 1,5-2 mg ermittelt. Weitere Dopaminagonisten wie Ropinirol, Lisurid und Dihydroergocryptin wurden ebenfalls in der Therapie des RLS und assoziierter PLMS erfolgreich eingesetzt. Die Zulassung für die Indikation RLS ist bislang lediglich für Ropinirol beantragt.

Neue Therapieansätze mit den Dopaminagonisten Rotigotine und Lisurid in Pflasterform sind wegen ihrer kontinuierlichen Wirkstofffreisetzung, ihrer überzeugenden Wirksamkeit und guten Verträglichkeit (Ausnahme Hautreaktionen an der Applikationsstelle) eine vielversprechende Therapieoption, die derzeit in klinischen Studien überprüft wird.

Der Einsatz von Dopaminagonisten bei der Therapie des RLS hat sich bewährt bei

- Auftreten von „Augmentation“ und „Time-shift“-Phänomenen unter L-Dopa-Therapie

- bei L-Dopa-Bedarf $>400 \mathrm{mg} / \mathrm{die}$

- bei schwerem RLS mit Beschwerden auch tagsüber.

Die Behandlung des RLS mit Dopaminagonisten verlangt eine kontinuierliche Dauertherapie, hier ist eine intermittierende oder „ad hoc“Medikation nicht anzuraten.

Wegen häufiger zu beobachtenden peripher-dopaminergen Nebenwirkungen wie arterieller Hypotonie, Übelkeit, Schwindel und Kopfschmerzen sollte die Dosis bei Therapiebeginn sehr langsam gesteigert werden und zumindest initial Domperidon ( $3 \times 2$ Tbl.) zusätzlich verordnet werden. Auch Tagesmüdigkeit, persistierende Schlafstörungen und Ödeme sind nicht seltene Nebenwirkungen einer Therapie mit Dopaminagonisten. Derzeit wird das Risiko ergothaltiger Dopaminagonisten wie Pergolid für Funktionsstörungen der Herzklappen sowie für pleuropulmonale und retroperitoneale Fibrosen diskutiert (13), weshalb insbesondere bei Risikopatienten unter Therapie mit ergothaltigen Dopaminagonisten eine kardiologische Überwachung angezeigt ist. 
Bei RLS-Patienten, die trotz dopaminerger Therapie noch über Einschlafstörungen klagen, nicht auf eine dopaminerge Therapie ansprechen oder Kontraindikationen für eine solche aufzeigen, können zur Behandlung des RLS auch Opiate oder Benzodiazepine eingesetzt werden.

\section{Opioide}

Obwohl es bislang in Deutschland noch keine klinischen Studien zur Wirksamkeit von Opioiden beim RLS gibt, hat sich in der klinischen Praxis der Einsatz von Tilidin/Naloxon (Valoron ${ }^{\circledR}$ ) bewährt. Um eine gleichmäßige Wirksamkeit zu gewährleisten, sollte eine Retard-Dosierung angestrebt werden, wobei die allgemeine Problematik der Opiattherapie auch bei RLS-Patienten beachtet werden muss.

\section{Andere Substanzen \\ der zweiten Wahl}

Schwer betroffene RLS-Patienten, die trotz dopaminerger Therapie keine ausreichende Beschwerdelinderung haben und über anhaltende Schlafstörungen klagen, benötigen oft eine Kombinationstherapie. Hier können neben Opioiden auch Benzodiazepine und Benzodiazepin-Rezeptor-Agonisten (z.B. Clonazepam, Zolpidem, Zopiclon) bzw. Antikonvulsiva (Gabapentin) hilfreich sein.

Nicht selten bedarf es einer geduldigen Differentialtherapie, in den meisten Fällen jedoch können die quälenden Symptome des RLS, die die Nächte der Betroffenen zum Alptraum machen, deutlich gelindert oder gar beseitigt werden. Allerdings handelt es sich bei allen angeführten Therapieformen um eine symptomatische Therapie, nach deren Absetzen die Beschwerden des RLS erneut auftreten.

\section{What is new in Restless Legs Syndrome?}

Dynamic process occurred in RLS research during the last years. An overview is provided on current status of epidemiology, pathophysiology, diagnostic approaches, classification as well as therapy of RLS. A prevalence of RLS in about $10 \%$ of the adult population in western countries is sta- bilized. New diagnostic criteria for $R L S$ and the development of standardized methods for diagnostic assessment of RLS and evaluation of its severity are of major importance. Recent clinical trials showed efficacy of $R L S$ therapy with dopaminergic drugs; however, treatment with $L$ dopa and some dopamine agonists can be hampered by augmentation. Alternative therapies are opioids and gabapentin.

\section{KeyWords:}

RLS - diagnostic criteria - therapy epidemiology - pathophysiology

\section{Literatur}

1. Allen RP, Earley CJ. Augmentation of the Restless Legs Syndrome with Carbidopa/Levodopa. Sleep 1996, 19: 205-213

2. Allen RP, Earley CJ. Restless legs syndrome: a review of clinical and pathophysiologic features. J Clin Neurophysiol. 2001; 18 : 128-147

3. Allen R, Picchietti D, Hening $W$ et al. Restless legs syndrome: diagnostic criteria, special considerations, and epidemiology. A report from the restless legs syndrome diagnosis and epidemiology workshop at the National Institute of Health. Sleep Medicine 2003; 4: 101-119

4. Benes $\mathrm{H}$, Heinrich $\mathrm{CR}$, Überall $M$, Kohnen R. Long-term safety and efficacy of cabergoline for the treatment of idiopathic Restless Legs Syndrome: Results from an open-label six-month clinical trial. Sleep 2004; 27: 674-682

5. Benes H, Kurella B, Kummer ], Kazenwadel J, Selzer R, Kohnen R. Rapid onset of action of Levodopa in restless legs syndrom: $A$ double blind, randomized, multicenter, crossover trial. Sleep 1999; 22: 1073-1081

6. Berger K, Luedemann J, Trenkwalder C, John U, Kessler $C$. Sex and the risk of restless legs syndrome in the general population. Arch Intern Med. 2004; 164: 196-202.

7. Collado-Seidel V, Kazenwadel J, Wetter TC, Kohnen R, Winkelmann J, Selzer R, Oertel WH, Trenkwalder C. A controlled study of additional sr-L-dopa in L-dopa responsive restless legs syndrom with late night symptoms. Neurology 1999; 52: 285-290

8. Dreykluft T, Karrasch J, Smuts J, Volc D, Grunstein R, Montplaisir J. The long-term safety and tolerability of ropinirole in RLS. Mov Disord 2004; 19 (Supplement 9): S430 (Abstract 1262)

9. The International Restless Legs Syndrome Study Group. Validation of the International Restless Legs Syndrome Study Group rating scale for restless legs. Sleep Medicine 2003; 4:121-132

10. Kohnen R, Oertel WH, Stiasny-Kolster K, Benes H, Trenkwalder C. Severity Rating of Restless Legs Syndrome: Validation of the RLS-6 scales. Sleep 2004; 27 (Supplement):A304 (Abstract 680)
11. Levchenko A, Montplaisir JY, Dube MP, Riviere JB, St-Onge J, Turecki G, Xiong L, Thibodeau P, Desautels A, Verlaan DJ, Rouleau $\mathrm{GA}$. The $14 \mathrm{q}$ restless legs syndrome locus in the French Canadian population. Ann Neurol 2004; 55: 887-891

12. Montplaisir J, Godbout R, Poirier G, Bédard MA. Restless legs syndrome and periodic movements in sleep: physiopathology and treatment with levodopa. Clin Neuropharmacol 1986; 9: 456-463

13. Rascol O, Pahak A, Bagheri H, Montastruc JL. New concerns about old drugs: vascular heart disease on ergot derivative dopamine agonists as an exemplary situation of pharmacovigilance. Mov Disord 204; 19 : 611-613

14. Rothdach AJ, Trenkwalder C, Haberstock J, Keil U, Berger K. Prevalence and risk factors of RLS in an elderly population. The MEMO Study. Neurology 2000; 54: 10641068

15. von Scheele C. Levodopa in restless legs. Lancet 1986; 23: 486-487

16. Stiasny K, Wetter TC, Winkelmann J, Brandenburg U, Penzel T, Rubin M, Hundemer HP, Oertel WH, Trenkwalder C. Longterm effects of pergolide in the treatment of restless legs syndrome. Neurology. 2001; 56:1399-1402

17. Stiasny K, Oertel WH, Trenkwalder C. Clinical symptomatology and treatment of restless legs syndrome and periodic limb movement disorder. Sleep Med Rev. 2002; 6: 253-265

18. Trenkwalder C, Stiasny K, Pollmächer Th, Schwarz J, Kohnen R, Kazenwadel J, Krüger HP, Ramm S, Künzel M, Oertel WH. L-Dopa-therapy of uremic and idiopathic restless legs syndrom: a double-blind crossover trial. Sleep 1995; 18: 681-688

19. Trenkwalder C, Collado Seidel V, Kazenwadel J, Wetter TC, Oertel W, Selzer R, Kohnen $R$. One-year treatment with standard and sustained-release levodopa: Appropriate long-term treatment of restless legs syndrome? Mov Disord. 2003; 18: 1184-1189

20. Winkelman JW, Johnston L. Augmentation and tolerance with long-term pramipexole treatment of restless legs syndrome (RLS). Sleep Med. 2004; 5: 9-14

21. Yasuda T. Nishimura A, Katsuki Y, Tsuji $Y$, Restless legs syndrome treated successfully by kidney transplantation - a case report. Clin Transpl 1986; 138: 138

\author{
Korrespondenzadresse: \\ Dr. med. Heike Beneš \\ Fachärztin für Neurologie und Psychiatrie, \\ Somnologin \\ somni bene \\ Institut für Medizinische Forschung und \\ Schlafmedizin Schwerin \\ Arsenalstr. 10 \\ 19053 Schwerin \\ E-Mail: heike.benes@somnibene.de
}

